\title{
Clinics in diagnostic imaging (176)
}

Chinthaka Appuhamy ${ }^{1}$, MBBS, MD, Justin Kwan ${ }^{1}$, MBBS, FRCR, Martin Weng Chin $\underline{H}^{\prime}$ g $^{1}$, MBBS, FRCR, Sriram Narayanan ${ }^{2}$, MBBS, FRCS, Sundeep Punamiya $^{1}$, MD, FSIR
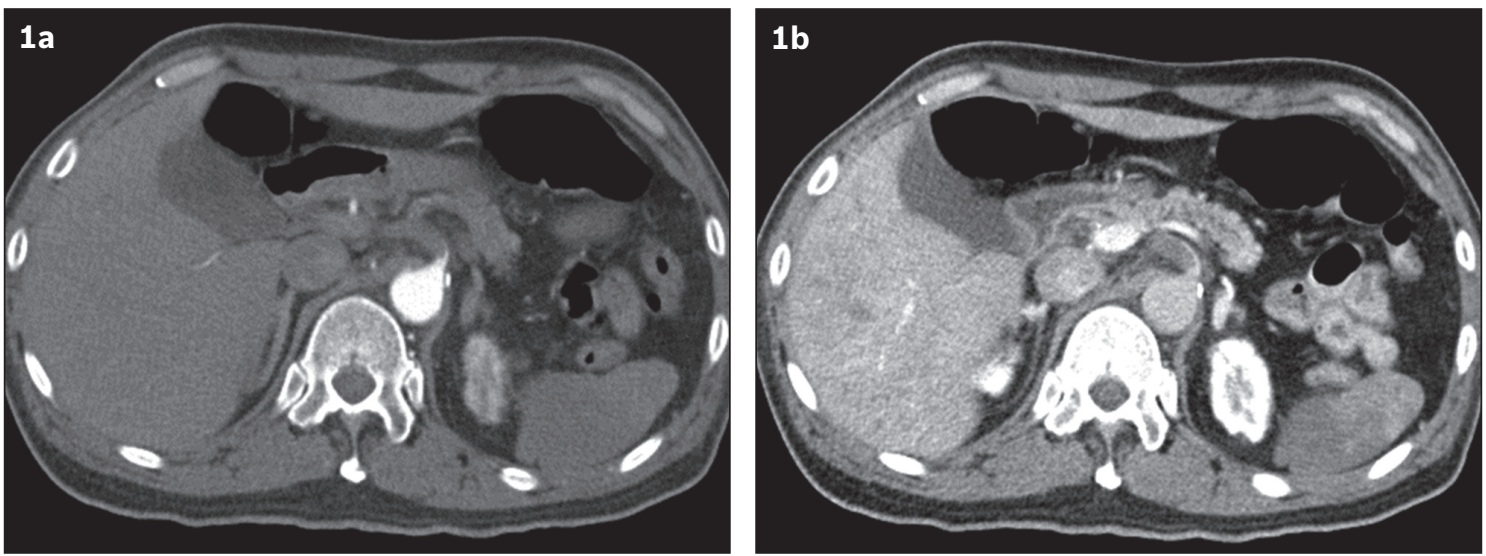

Fig. 1 Axial CT images in (a) arterial and (b) delayed phases through the upper abdomen

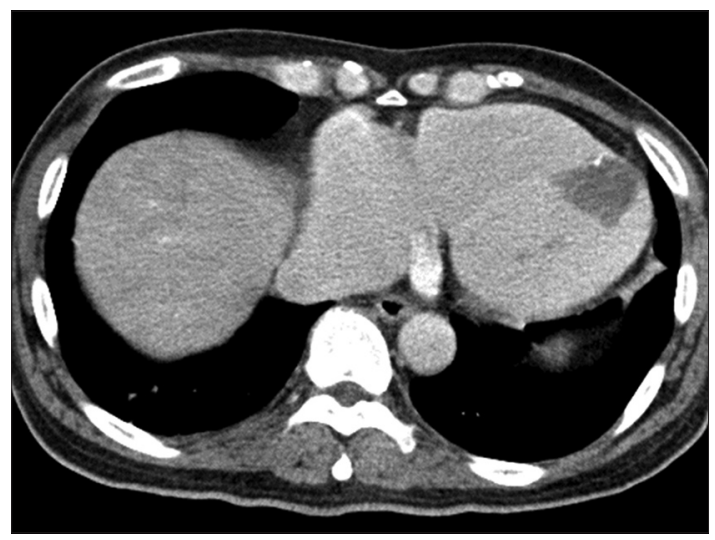

Fig. 2 Axial CT image at the level of the heart.

\section{CASE PRESENTATION}

A 52-year-old man presented to the emergency department with progressively worsening upper abdominal pain of one week's duration. He was a heavy smoker with a history of chronic heart disease and atrial fibrillation, but was not compliant with his warfarin therapy. An echocardiogram performed two months before had shown a thrombus in the left ventricular apex and left atrial appendage.

The patient's vital signs included a heart rate of 122 beats per minute (irregularly irregular), blood pressure of 123/68 mmHg, respiratory rate of 20 breaths per minute and oxygen saturation of $99 \%$ on room air. He was afebrile, alert and showed no signs of distress. Pertinent physical findings included a soft abdomen with moderate tenderness elicited in the epigastrium and left hypochondrium. There was no rebound tenderness, guarding or

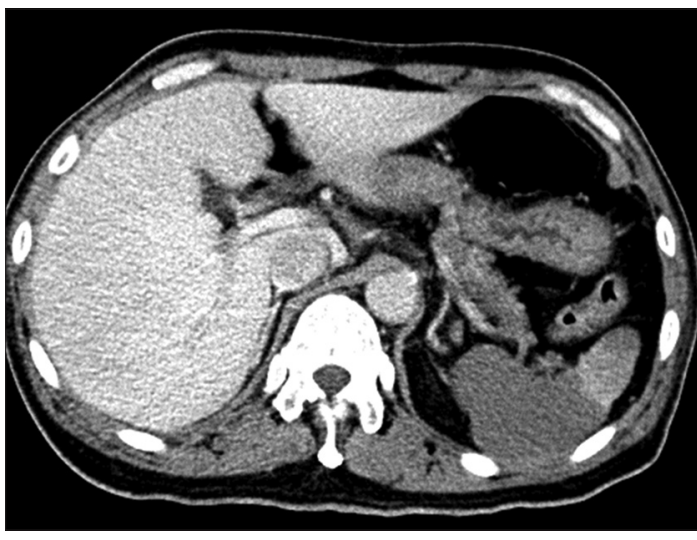

Fig. 3 Axial CT image through the upper abdomen.

abdominal distension. Bowel sounds were present. The remainder of the physical examination was non-contributory. Bedsidefocused ultrasonography showed no evidence of free fluid.

Initial laboratory results showed a mildly raised white blood cell count of $10.4 \times 10^{9} / \mathrm{L}$ and serum lactate level of $2.4 \mathrm{mmol} / \mathrm{L}$ (normal range $0.5-2.2 \mathrm{mmol} / \mathrm{L}$ ). The patient's liver function test results were mostly within normal limits (alanine aminotransferase $12 \mathrm{U} / \mathrm{L}$, aspartate aminotransferase $21 \mathrm{U} / \mathrm{L}$, bilirubin $24 \mu \mathrm{mol} / \mathrm{L}$, alkaline phosphatase $97 \mathrm{U} / \mathrm{L}$ ), with the exception of mildly raised gamma-glutamyl transpeptidase (GGT) (61 U/L, normal range $7-50 \mathrm{U} / \mathrm{L})$. His international normalised ratio was normal, measuring 1.2.

Contrast-enhanced computed tomography (CT) of the abdomen was performed shortly after the patient was admitted. What do the CT images (Figs. 1-3) show? What is the diagnosis?

${ }^{1}$ Department of Diagnostic Radiology, ${ }^{2}$ Department of Vascular Surgery, Tan Tock Seng Hospital, Singapore

Correspondence: Dr Justin Kwan, Associate Consultant, Department of Diagnostic Radiology, Tan Tock Seng Hospital, 11 Jalan Tan Tock Seng, Singapore 308433. justinkwan07@gmail.com 


\section{IMAGE INTERPRETATION}

Axial CT images in the arterial (Fig. 1a) and delayed (Fig. 1b) phases show complete occlusion of the coeliac artery, common hepatic artery and splenic artery. Axial CT image at the level of the heart (Fig. 2) shows an eccentric mural thrombus within the apex of the left ventricle. Axial CT image taken at the level of the spleen (Fig. 3) shows persistent non-enhancement of a segment of the spleen, compatible with a splenic infarct. The superior mesenteric artery (SMA) and the gastroduodenal artery (GDA) are patent. The liver shows normal perfusion without any evidence of hepatic ischaemia. There was no evidence of gastric wall thickening or perforation.

\section{DIAGNOSIS}

Acute embolic occlusion of the coeliac artery.

\section{CLINICAL COURSE}

In view of the $\mathrm{CT}$ findings and progressively worsening abdominal pain, the decision was made to proceed with endovascular treatment of the coeliac artery after excluding any contraindication to thrombolysis. The right common femoral artery was punctured and an 8 French ( Fr) vascular sheath (Terumo Medical Corporation, Tokyo, Japan) was inserted. A 5 Fr Simmons 1 (Terumo Medical Corporation, Tokyo, Japan) was used to cannulate the coeliac artery co-axially through a $7 \mathrm{Fr}$ RDC (Vista Brite Tip; Cordis, Miami, FL, USA) guiding catheter (Fig. 4). The occlusion was easily crossed using a 0.035-inch glidewire (Terumo Medical Corporation, Tokyo, Japan), suggesting the acute nature of the occlusion. A $5 \mathrm{Fr} \times 10 \mathrm{~cm}$ Fountain infusion catheter (Merit Medical, Galway, Ireland) was placed across the occluded segment of the coeliac artery. A bolus of $15 \mathrm{mg}$ of recombinant human tissue-type plasminogen activator (rtPA) (Alteplase, Boehringer Ingelheim, Ingelheim, Germany) was given over 20 minutes with another $6 \mathrm{mg}$ infused over the next 12 hours. Additional intravenous heparin was infused at $200 \mathrm{IU} / \mathrm{hr}$.

During the course of the thrombolysis infusion, the patient's abdominal pain had markedly improved, with a downtrending serum lactate level $(2.0 \mathrm{U} / \mathrm{L})$ and normalisation of the GGT level $(50 \mathrm{U} / \mathrm{L})$. The rest of the liver transaminases remained within normal limits. A subsequent follow-up angiogram at 12 hours (Fig. 5) showed complete dissolution of the embolus with restoration of flow within the coeliac artery. The splenic artery remained occluded. Slow flow was noted in the right main hepatic artery, suggestive of distal migration of the clot. Distally, however, flow was still seen in the right intrahepatic branches from the intrahepatic collateral vessels. The left hepatic artery and its branches, as well as the GDA and main portal vein, were patent.

In view of the presence of intrahepatic collateral flow in the right hepatic lobe, as well as improving clinical symptoms and lab results, it was thought unnecessary to further treat the migrated clot in the right main hepatic artery. Hence, the rtPA was discontinued, and the infusion and guiding catheters were removed. The groin sheath was removed two days later. The
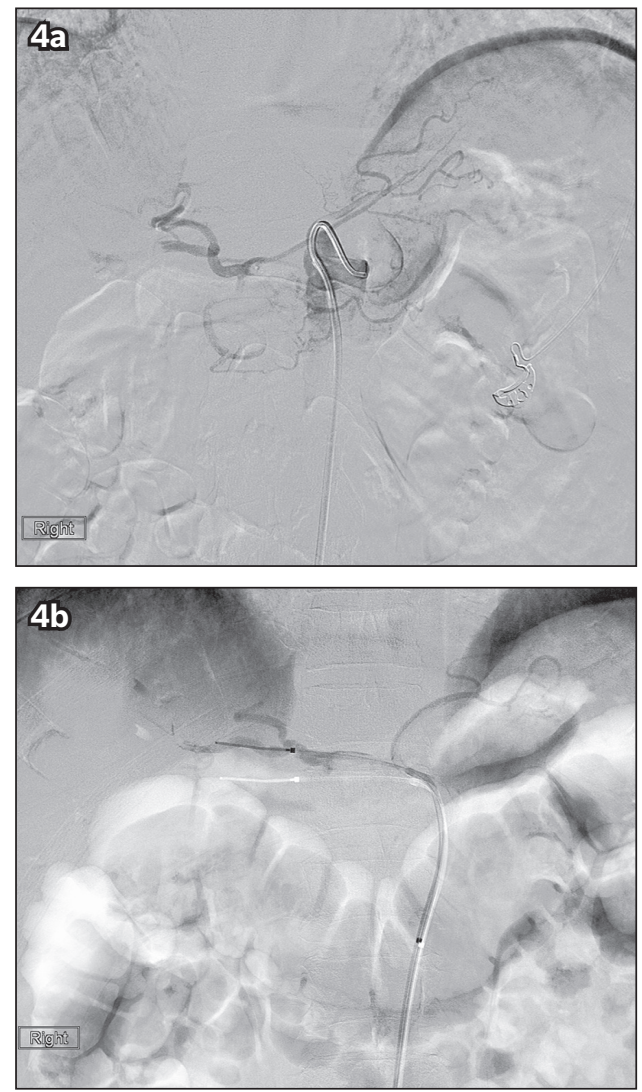

Fig. 4 (a) Selective angiogram of the coeliac artery shows a filling defect within, extending into the common hepatic and splenic arteries; distal opacification of the hepatic artery proper occurs via retrograde flow through the superior mesenteric artery and gastroduodenal artery. (b) Angiogram of the coeliac artery shows that the guiding catheter was placed in the ostium of the coeliac artery with the thrombolysis infusion catheter positioned across the occluded segment.

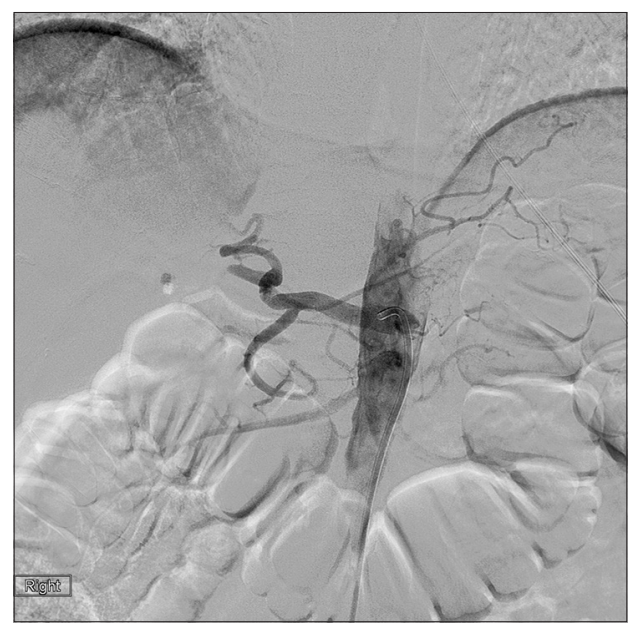

Fig. 5 Repeat angiogram via the existing thrombolysis infusion catheter (not shown) and guiding catheter at 12 hours shows resolution of the thrombus with re-established flow within the coeliac artery. The splenic artery is not opacified and remains occluded.

patient remained asymptomatic in the ward and was subsequently discharged on long-term oral anticoagulation therapy. At his clinic review six weeks after discharge, the patient was well with no further abdominal pain, and his liver transaminase and serum lactate levels were normal. 

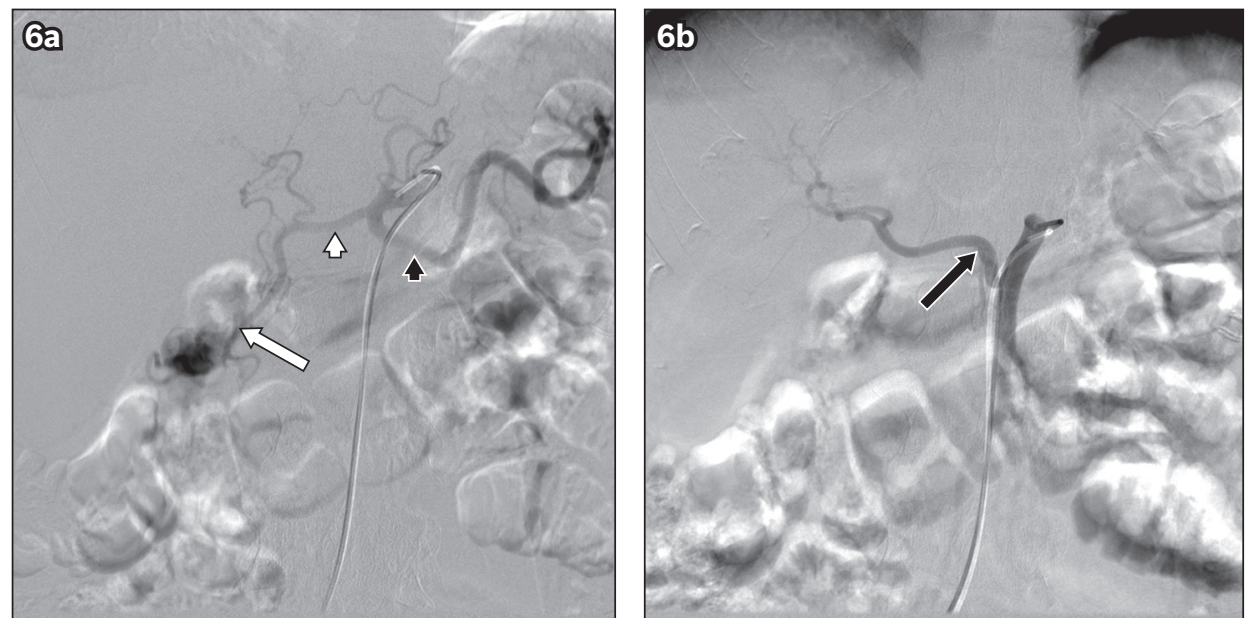

Fig. 6 An elderly patient presented with massive upper gastrointestinal bleeding. (a) On selective cannulation of the coeliac artery, catheter angiogram shows opacification of the common hepatic artery (white arrowhead), splenic artery (black arrowhead) and gastroduodenal artery (white arrow). The right hepatic artery is not seen. (b) After cannulating the superior mesenteric artery (SMA), catheter angiogram shows a replaced right hepatic artery (black arrow) arising from the proximal SMA.

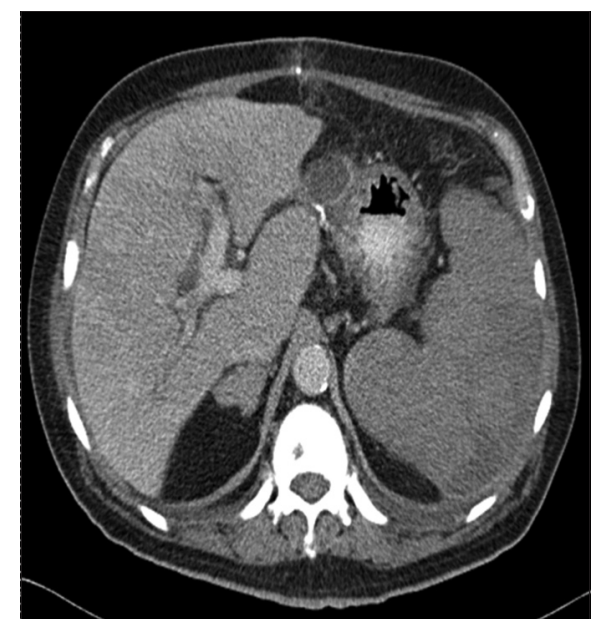

Fig. 7 A middle-aged man presented with upper abdominal pain and sepsis. Axial CT image shows marked enlargement of the liver and spleen with patchy areas of hypoperfusion, suspicious for hepatic and splenic infarction.

\section{DISCUSSION}

Acute coeliac artery occlusion, a rare cause of acute abdominal pain, has high mortality and morbidity when diagnosis and treatment are delayed. Besides cardiac disorders, such as atrial fibrillation, which can result in occlusion of virtually any artery, other causes that have been described in the literature by authors such as Tanaka et al, ${ }^{(1)}$ Trellopoulos et al ${ }^{(2)}$ and Arul et al included:(3) acute pancreatitis; recent upper gastrointestinal surgery; blood dyscrasias (e.g. antiphospholipid syndrome or thrombophilia); structural abnormalities such as compression by the median arcuate ligament; and oral contraceptive drugs. Most of these occlusions remain asymptomatic due to their gradual development as well as the vast collateral circulation from the SMA. ${ }^{(4)}$ However, in cases of acute embolism, visceral and endorgan ischaemia may result in vague symptoms such as persistent abdominal pain, nausea and vomiting.

In cases of splenic infarction, which may occur in various clinical conditions, pain usually resolves gradually without clinical sequelae and surgery is required only when complications such as infection arise. ${ }^{(5)}$ Gastric ischaemia is uncommon, as the stomach draws a rich blood supply from branches of both the coeliac artery and SMA collaterals, although one case report from Kelekis et al described gastric perforation secondary to atherosclerotic occlusion of both vessels. ${ }^{(6)}$ Hepatic ischaemia is also uncommon, as the liver has a dual blood supply from both the hepatic artery and the portal venous system.

Imaging plays a crucial role when evaluating patients with suspected coeliac artery occlusion. Radiographs of the abdomen are usually unremarkable unless there are features of intestinal obstruction or perforation. Mesenteric CT angiography is accurate in depicting acute coeliac artery occlusion and its potential complications. A study of 26 patients showed that biphasic CT with mesenteric CT angiography was $100 \%$ specific, but only $73 \%$ sensitive in the diagnosis of coeliac artery, SMA and inferior mesenteric artery occlusion. ${ }^{(7)}$ Intraluminal hypodensity within the coeliac artery, with non-enhancement of the distal vessels, would confirm the presence of an occluding thrombus or embolus. CT has the additional benefit of being able to detect anatomical variants such as a replaced right hepatic artery arising from the SMA, which may be crucial when planning for endovascular treatment (Fig. 6). Complications such as ischaemia or infarction would also be visible in cases where there is absence of or delayed enhancement of the involved organ (Fig. 7).

Angiography still remains the gold standard in the diagnosis of coeliac artery occlusion. ${ }^{(8)}$ It has the additional benefit of supplying temporal information that allows the assessment of any collateral flow supplied from branches of the SMA. However, this imaging technique may not be suitable for all patients in the acute setting due to possible contraindications to catheter angiography, such as underlying coagulopathy or renal impairment.

Treatment options for acute coeliac artery occlusion vary between different centres. Possible options include endovascular treatment with catheter-directed intra-arterial thrombolysis, mechanical thrombectomy, systemic anticoagulation and open surgery. While endovascular treatment is infrequently described in the literature, it has been shown to have good results with a 
low incidence of complications, lower morbidity and a high technical success rate. ${ }^{(9)}$

In summary, isolated acute coeliac artery occlusion is a rare occurrence in patients with atrial fibrillation; when present, it can produce significant symptoms and result in end-organ infarction. Thorough preoperative imaging, including CT angiography, is very useful in assessing vascular involvement of the mesenteric vessels, as well as any potential complications. Catheter-directed intra-arterial thrombolysis is a feasible and effective method of treating acute thromboembolic occlusion of the coeliac artery.

ABSTRACT A 52-year-old man, who had a background of chronic heart disease and atrial fibrillation, as well as non-compliance with warfarin therapy, presented with a two-week history of worsening upper abdominal pain. Computed tomography mesenteric angiography showed complete embolic occlusion of the coeliac artery with resultant segmental splenic infarction, and thrombus within the left ventricle. A decision was made to proceed with catheter-directed thrombolysis. Subsequent followup angiogram at 12 hours showed successful treatment with complete dissolution of the coeliac embolus. The patient's symptoms resolved during his hospitalisation and he was subsequently discharged well on long-term oral anticoagulation therapy. Isolated acute embolic occlusion of the coeliac axis is a rare occurrence that may result in end-organ infarction. Treatment options include systemic anti-coagulation, mechanical thrombectomy, catheter thrombolysis or open surgery. Catheter-directed thrombolysis therapy is a feasible and effective option for treating acute thromboembolic occlusion of the coeliac artery.

Keywords: atrial fibrillation, catheter-directed thrombolysis, coeliac artery occlusion, embolus

\section{REFERENCES}

1. Tanaka Y, Nakajima M, Hirano T, Uchino M. Cardioembolic stroke followed by isolated celiac artery thromboembolism. Intern Med 2007; 46:1463-6.

2. Trellopoulos G, Pikilidou MI, Tsiga E. Celiac artery embolism due to thrombophilia - a case report. Int J Angiol 2009; 18:96-8.

3. Arul GS, Dolan G, Rance CH, Singh SJ, Sommers J. Coeliac axis thrombosis associated with the combined oral contraceptive pill: a rare cause of an acute abdomen. Pediatr Surg Int 1998; 13:285-7.

4. Farma JM, Hoffman JP. Nonneoplastic celiac axis occlusion in patients undergoing pancreaticoduodenectomy. Am J Surg 2007; 193:341-4; discussion 344.

5. O'Keefe JH Jr, Holmes DR Jr, Schaff HV, Sheedy PF 2nd, Edwards WD. Thromboembolic splenic infarction. Mayo Clin Proc 1986; 61:967-72.

6. Kelekis NL, Athanassiou E, Loggitsi D, et al. Acute occlusion of the celiac axis and its branches with perforation of gastric fundus and splenic infarction, findings on spiral computed tomography: a case report. Cases J 2010; 3:82.

7. Kirkpatrick ID, Kroeker MA, Greenberg HM. Biphasic CT with mesenteric CT angiography in the evaluation of acute mesenteric ischemia: initial experience. Radiology 2003; 229:91-8.

8. Patten RM, Coldwell DM, Ben-Menachem Y. Ligamentous compression of the celiac axis: CT findings in five patients. AJR Am J Roentgenol 1991; 156:1101-3

9. Mohammed A, Teo NB, Pickford IR, Moss JG. Percutaneous transluminal angioplasty and stenting of coeliac artery stenosis in the treatment of mesenteric angina: a case report and review of therapeutic options. J R Coll Surg Edinb 2000; 45:403-7. 


\section{SINGAPORE MEDICAL COUNCIL CATEGORY 3B CME PROGRAMME} (Code SMJ 201704B)

Question 1. Regarding acute coeliac artery occlusion:

(a) It is a common cause of abdominal pain.

(b) It can be easily diagnosed clinically, as patients present with typical symptoms.

(c) It can be life-threatening if left untreated.

(d) It is best diagnosed on Doppler ultrasonography.

Question 2. Regarding gastric ischaemia:

(a) This entity commonly occurs in patients with abdominal pain.

(b) The stomach is supplied by branches of the coeliac axis.

(c) The stomach does not receive any supply from the superior mesenteric artery.

(d) Gastric perforation may occur.

Question 3. Common complications of acute coeliac artery occlusion include:

(a) Hepatic ischaemia.

(b) Gastric ischaemia and perforation.

(c) Splenic infarction.

(d) Acute pancreatitis.

Question 4. Regarding imaging in coeliac artery occlusion:

(a) Radiographs may show features of intestinal perforation.

(b) Computed tomography $(\mathrm{CT})$ can detect anatomic variants of hepatic arteries.

(c) Mesenteric CT angiography cannot be used to visualise the complications of coeliac artery occlusion.

(d) There is currently no role for angiography in the diagnosis of coeliac artery occlusion.

Question 5. Treatment options for acute coeliac artery occlusion include:

(a) Endovascular catheter-directed thrombolysis therapy.

(b) Intra-arterial gelfoam infusion.

(c) Oral anticoagulation treatment.

(d) Open surgery.

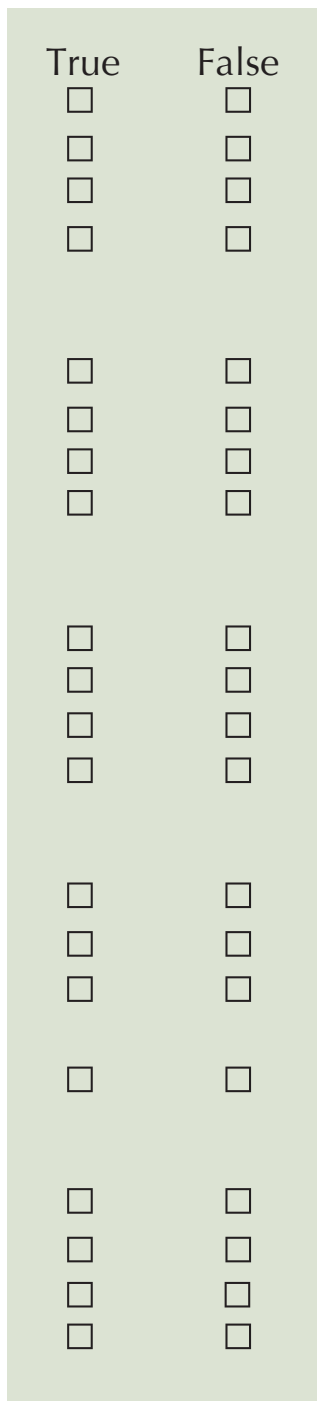

\section{Doctor's particulars:}

Name in full

MCR number

Specialty:

Email address

\section{SUBMISSION INSTRUCTIONS:}

(1) Visit the SMJ website: http://www.smj.org.sg/current-issue and select the appropriate set of questions. (2) Provide your name, email address and MCR number. (3) Select your answers and click "Submit".

\section{RESULTS:}

(1) Answers will be published online in the SMJ June 2017 issue. (2) The MCR numbers of successful candidates will be posted online at the SMJ website by 31 May 2017. (3) Passing mark is $60 \%$. No mark will be deducted for incorrect answers. (4) The SMJ editorial office will submit the list of successful candidates to the Singapore Medical Council. (5) One CME point is awarded for successful candidates.

Deadline for submission: (April 2017 SMJ 3B CME programme): 12 noon, 24 May 2017. 